\title{
LA FORMACIÓN EN CULTURA FÍSICA Y DEPORTE: ESTRATEGIA PARA UNA EDUCACIÓN DE CALIDAD EN LA UNESUM
}

AUTORES: Rubén Darío Acebo Calderón ${ }^{1}$

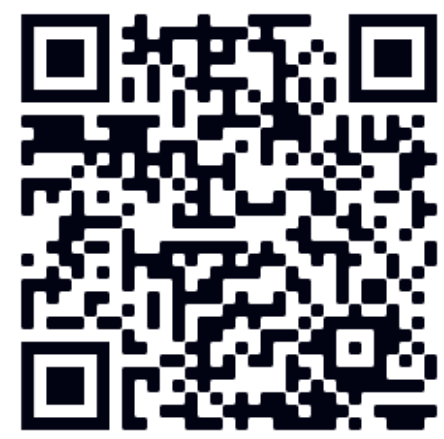

DIRECCIÓN PARA CORRESPONDENCIA: Ruben.Dario@unsum.edu.ec

Fecha de recepción: 12/06/2019

Fecha de aceptación: 20/07/2019

\section{RESUMEN}

La cultura Física es el trato y convivencia con los estudiantes y nos revela hoy las debilidades éticas y profesionales, en la cual la educación superior debe actuar de manera urgente. El presente artículo tiene la finalidad de hacer los fundamentos de la relación entre la formación estudiantil, en la cultura física y el deporte, sugerir la estrategia pedagógica que se aplica en el tratamiento colectivo al estudiante universitario de las diferentes Facultades en la Cultura Física de la universidad Estatal del Sur de Manabí, la cual centra su atención en la cultura recreativa deportiva, con el fin de contribuir a la formación profesional e integral de los estudiantes universitarios.

PALABRAS CLAVE: cultura del trato estudiantil, cultura histórica deportiva, educación estudiantil.

\section{TRAINING IN PHYSICAL CULTURE AND SPORTS: STRATEGY FOR A QUALITY EDUCATION IN THE UNESUM}

\section{ABSTRACT}

Physical culture is the treatment and coexistence with students and today reveals ethical and professional weaknesses, in which higher education must act urgently. This article aims to make the fundamentals of the relationship between student training, physical culture and sports, suggest the pedagogical strategy that is applied in the collective treatment of university students of the different Faculties in the Physical Culture of the State University of the South of Manabí, which focuses on recreational sports culture, in order to contribute to the professional and comprehensive training of university students

KEYWORDS: Culture of student treatment, historical sports culture, student education.

\footnotetext{
${ }^{1}$ Universidad Estatal del Sur de Manabí, departamento de Cultura Física y Deporte, jipijapa- Manabí- Ecuador
} 
Rubén Darío Acebo Calderón

\section{INTRODUCCIÓN}

Una esencial enseñanza con pedagogía educacional, es lograr la preparación de los estudiantes como profesionales, capaces de actuar con la sociedad, dando un buen sentido de pertenencia, dignidad y patriotismo. Es así que la cultura deportiva espiritual universitaria sedimente los estrechos vínculos entre el deporte, la sociedad y la universidad en el buen vivir.

Reconociendo que se ha logrado la transformación necesaria en actuación de los docentes y estudiantes según las normas de comportamiento estudiantil y se contribuyó a los diferentes niveles de enseñanza en la formación del estudiantado, culto, sano, portador de una cultura de paz y apto para el trabajo útil en la sociedad; capaz de actuar de manera activa y adecuada en las transformaciones sociales. La educación superior tiene un papel importante en este método que implementan los centros de cultura física y deportes, apoyados por el Ministerio de Educación Superior.

Estos pilares de la universidad del siglo XXI, defiende la necesidad de promover en sus profesionales y estudiantes la condición de ser, saber, saber hacer, saber convivir, y de reforzar el comportamiento estudiantil. Los centros universitarios pueden responder con el desarrollo de la cultura histórica deportiva en el proceso formativo, amparado este en la amplitud de matices e impactos de las actividades físico-deportivas y recreativas en la sociedad.

La Universidad tiene la Ciencias de la Cultura Física y el Deporte debe fortalecer de manera creativa estilos de trabajo adecuados para continuar educando individuos altamente comprometidos con la humanidad, dispuestos a propiciar riquezas materiales y espirituales, fomentar prácticas saludables y mejorar la calidad de vida de la estudiantil universitaria.

La cultura física ha estado entre los objetivos y estrategias educativas. A partir de estos presupuestos en el 2001 en la UNESUM. Y se determinaron las bases de la formación integral a través de proyectos educativos multidimensionales. A pesar de ello, no se ha logrado armonizar los contenidos históricos con el objeto de la profesión de manera contextualizada en su dialéctica e interrelación. Aún se descuida la cultura histórica deportiva en la preparación ética y socio profesional.

El significar de la cultura histórica deportiva -desde el valor de las glorias del deporte como patrones conductuales- en la memoria histórica del patrimonio de la cultura ciudadana y pedagógica favorece la calidad del proceso de formación profesional y social de los estudiantes universitarios en general y, especialmente, de aquellos vinculados a la cultura física y los deportes.

\section{DESARROLLO}

Dentro de la Estrategia Nacional de Educación Ambiental uno delos objetivos esenciales es fomentar la educación ambiental como parte de la formación integral del estudiantado de los niveles de Educación Inicial, General Básica y Bachillerato para impulsar metodologías, técnicas y recursos de educación y comunicación ambiental, que faciliten el desarrollo de conocimientos, comportamientos y prácticas socio-ambientales que estimulen una relación positiva y coherente entre el ser humano, integrando los saberes y ciencias de los diversos pueblos y nacionalidades del Ecuador (Ministerio del Ambiente, 2019)

El proceso de investigación cumplió con esta estrategia nacional, está relacionado con la evaluación de las actividades relacionadas con el manejo de desechos sólidos de los estudiantes para mejorar 
el cuidado del medio ambiente en las instituciones docentes

es un estudio cualitativo, la metodología utilizada es descriptiva, y acción-participativa.

En este proceso investigativo sobre una realidad social, se hace una descripción del objeto de estudio y de sus factores implícitos, para realizar un análisis de esa cualificación realizada, para así tener un acercamiento a la realidad de esta problemática ambiental; que está sujeto a las acciones por parte de los docentes ejecutores esta investigación, en cuanto a la realización de las observaciones y encuentros con los estudiantes y la comunidad educativa que hacen parte del proceso evolutivo del proyecto, participando en su desarrollo.

Para el desarrollo de este trabajo de investigación se pudo obtener la información necesaria para describir el problema del manejo de los desechos sólidos y su relación con el cuidado del medio ambiente; en la decisión sobre el mejor modo de obtener los datos y de quién o quiénes obtenerlos. se toman en el campo, ya que los participantes del estudio nos resultan desconocidos cuando se inicia este proceso y es la propia información obtenida la que va guiando el muestreo y el diagnóstico de la realidad en el contexto estudiado (Sampieri, 2018).

En una primera etapa se realizó la búsqueda de información secundaria, por medio de una revisión bibliográfica y documental, a efectos de revisar los principales referentes conceptuales relacionados con la temática de investigación.

La metodología empleada en la investigación corresponde al tipo exploratorio, que ayuda a familiarizar a los estudiantes con el contexto de la problemática, en este caso el sector de la ciudad de Jipijapa y a partir de ello recopilar información específica sobre las principales características del entorno (Sampieri, 2018).

Cabe destacar que la recopilación de información primaria, que complementa la información de las fuentes secundarias se realizó por medio de la observación no participante y la aplicación de dos técnicas de investigación con fin de recolectar información más concreta: una encuesta dirigida a los moradores de la comunidad, y una entrevista en la que se formuló a los dirigentes una serie de preguntas referentes al tema estudiado (Sampieri, 2018).

En el proceso de la investigación se realizó un diagnóstico situacional del sector, utilizando fichas de observación, encuestas, entrevistas para establecer la realidad educativa ambiental y el manejo de los residuos sólidos manejo de los residuos sólidos. Este sistema de actividades educativas permite disminuir la contaminación de la naturaleza y mejorar la imagen de las instituciones educativas y la comunidad.

En esta investigación se comprobó que en el centro si existía un programa que norma o estableciera las actividades a realizar en el plano medio ambiental, el director de dicho centro respondió, que no existe, o sea, una planificación para ejecutar actividades.

Se aplico una encuesta a los estudiantes de la escuela Judith Iza de Antón, donde se realizó el estudio de campo, se aplicó encuesta de preguntas abiertas y cerradas que proporcionaron la información necesaria, para diagnosticar las necesidades de planificar, ejecutar y evaluar actividades relacionadas con el manejo de desechos sólidos y su relación con la educación ambiental en la institución educativa.

La primera parte (diagnóstico inicial) que trata sobre el conocimiento del manejo medio ambiente, como lo definen y que problemas de su municipio conocen. En la segunda parte, se indagó si realizan actividades de educación ambiental en las clases de Educación Física y actividades físicas extradocentes y extraescolares o la disposición a realizar actividades de este tipo. 
Rubén Darío Acebo Calderón

La población está formada 230 estudiantes y 20 docentes, de la institución educativa su función básica es determinar el estudio de las variables en dicha institución. El muestreo realizado es probabilístico (aleatorio): fueron muestreados 70 estudiantes de nivel básico superior y sus 10 docentes de la sesión vespertina, en este muestreo todos los integrantes de la población pueden formar parte de la muestra, tienen probabilidad positiva de formar parte de la muestra, por lo tanto, es el tipo de muestreo a utilizar en el proceso de investigación, por ser el riguroso y científico (Sampieri, 2018).

Existió una relación directa entre los parámetros estudiados en el proceso de evaluación sobre: sobre lo conocimiento-información y sensibilización-motivación. Se observó trabajo en equipo disciplinario, metódico, coherente y responsable en el proceso de búsqueda y selección de la información que permitieron comprender de manera clara y precisa la problemática estudiada y sensibilizar su participación humana en disminuir el deterioro de los recursos naturales y a generar motivación para encontrar soluciones a la misma.

Se desarrolló las capacidades de experimentación e interacción, que determinó que la actividad ejecutada facilitó la exteriorización de los pensamientos, criterios, necesidades, sugerencias e ideas que les permitieron integrarse en el trabajo; asimilar de manera personal la información obtenida para planificar, diseñar y experimentar diferentes actividades; seguir las directrices del docente guía y del líder del grupo; y, fortalecer la capacidad para generar soluciones viables ante la problemática ambiental analizada, sobre la base del paradigma constructivista del aprendizaje, donde los estudiantes guiados por el docente es capaz de hacer y de forma creativa aplicar estas actividades en la práctica para mejorar el manejo de los desechos sólidos y con ello el cuidado del medio ambiente (Carranza, 2005).

Se logró la valoración-compromiso y acción voluntaria-participación que se manifestó en el fortalecimiento de la relación docente - estudiante, se mantuvo un compromiso permanente para alcanzar la meta propuesta fundamentada en la participación voluntaria.

Se valoró la expresión práctica de la capacidad humana para corregir errores y la necesidad de generar cultura, responsabilidad y ética ambiental a nivel individual, comunitario en el cuidado del medio ambiente en los estudiantes.

Se realizó una jornada de concientización ambiental sobre el manejo adecuado de los residuos sólidos y la aplicación de una encuestas a la muestra seleccionada donde se evidencia el nivel de conocimiento que tienen sobre el manejo de los residuos sólidos, constatando el índice cognitivo sobre el impacto ambiental que genera el mal uso de los residuos sólidos.

Se fortaleció el rol docente en el acto de educar y concienciar a las diferentes generaciones para desarrollar competencias éticas y reorientar las prácticas de los grupos, al reconocer en la interacción maestro/estudiante un ejercicio de construcción de significados compartidos acerca de la educación ambiental orientada hacia una conciencia ambiental, además de su incidencia en las formas de valorar y actuar frente al ambiente, en una reinvención de relaciones más armónicas entre las comunidades y de estas con el entorno.

\section{CONCLUSIONES}

La observación a la institución y a su entorno determinó el impacto visual negativo que produce el mal uso de los residuos sólidos en la institución, arrojando así un diagnóstico de gran utilidad a la presente investigación pues permiten planificar, ejecutar y evaluar las actividades en la institución educativa. 
Se identificó el interés y compromiso entre el docente-estudiante durante el proceso de planificación y ejecución de las actividades educativas para mejorar la manipulación de los desechos sólidos para proteger el medio ambiente de las instituciones y la comunidad.

Los estudiantes condujeron adecuadamente la selección y recopilación bibliográfica acorde a la necesidad, se demostró respeto y cumplimiento a las disposiciones, se promovió la investigación (causa-efecto) de la problemática y el análisis metódico a nivel cualitativo y cuantitativo para definir soluciones.

Se demostró capacidad de liderazgo, trabajo en equipo y predisposición en la socialización de la información durante la realización de los resultados de las actividades realizadas para promover cultura, ética y responsabilidad ambiental en los docentes y estudiante a nivel institucional y de las unidades educativas participantes.

La educación ambiental puede contribuir al manejo de los desechos sólidos y puede generar nuevos comportamientos, actitudes, valores y creencias que impulsen el desarrollo social, productivo y creador; como consecuencia puede ser el medio para el logro de nuevas relaciones entre los seres humanos y esencialmente en la formación de las nuevas generaciones, responsables del cuidado de este proceso en el futuro.

\section{REFERÉNCIAS BIBLIOGRÁFICAS}

Explora Natura . (24 de mayo de 2019). Interdisciplinariedad Educación Ambiental. Obtenido de https://www.exploranatura.com/interdisciplinariedad-educacion-ambiental-2/

Arichabala. (2014). Los 10 Mejores Proyectos sobre el cuidado del medio ambiente y el manejo de desechos sólidos. Quito: ODEBRECHT.

Cadmea, M., \& et al. (2015). La Educación Ambiental en la Educación Superior. Quito: Facultad de Ingenieria Ambiental y recursos naturales.

Carranza, R. (2005). El constructivismo como estrategia educativa:formación profesional . Ensayo, 5-12.

Follari, R. (2001). Estudios culturales, transdisciplinariedad e interdisciplinariedad. Unidad y Praxis.

Martínez, \& Balaguer. (2016). Universidad saludable: una estrategia de promoción de la salud y salud en todas las políticas para crear un entorno de trabajo saludable. Barcelona: Arch Prev Riesgos Labor vol.19 no.3.

Ministerio del Ambiente. (junio de 24 de 2019). Dirección de Información, Seguimiento y Evaluación -DISE. Obtenido de maeduca@ambiente.gob.ec

Plan Nacional de Desarrollo. (2017-2021). Sobre los aprendizajes compartidos: Presencia e iniciativas de las instituciones a favor de la educación ambiental. Quito: Educación para el desarrollo sostenible.

Sampieri. (2018). Metodología de la investigación: las rutas cuantitativa, cualitativa y mixta. Mexico: McGraw Hill Mexico. 
